\title{
GOOD WEIGHTS FOR WEIGHTED CONVOLUTION ALGEBRAS
}

\author{
SANDY GRABINER \\ Department of Mathematics, Pomona College \\ 610 N. College Avenue, Claremont, CA 91711, U.S.A. \\ E-mail:sgrabiner@pomona.edu
}

\begin{abstract}
Weighted convolution algebras $L^{1}(\omega)$ on $\mathbf{R}^{+}=[0, \infty)$ have been studied for many years. At first results were proved for continuous weights; and then it was shown that all such results would also hold for properly normalized right continuous weights. For measurable weights, it was shown that one could construct a properly normalized right continuous weight $\omega^{\prime}$ with $L^{1}\left(\omega^{\prime}\right)=L^{1}(\omega)$ with an equivalent norm. Thus all algebraic and norm-topology results remained true for measurable weights. We now show that, with careful definitions, the same is true for the weak* topology on the space of measures that is the dual of the space of continuous functions $C_{0}(1 / \omega)$. We give the new result and a survey of the older results, with several improved statements and/or proofs of theorems.
\end{abstract}

1. Introduction. Our goal is to show that all weighted convolution algebras on $\mathbf{R}^{+}=$ $[0, \infty)$ have essentially the same tools available that are used to study $L^{1}\left(\mathbf{R}^{+}\right)$. For us, a weight is a positive Borel function $\omega$ on $\mathbf{R}^{+}$which is bounded and bounded away from 0 on all intervals $[0, b] . L^{1}(\omega)$ is the Banach space of (equivalence classes of) locally integrable functions on $\mathbf{R}^{+}$for which the norm $\|f\|=\|f\|_{\omega}=\int|f(t)| \omega(t)$ is finite. In a similar way, $M(\omega)$ is the Banach space of locally finite complex Borel measures $\mu$ for which the norm $\|\mu\|=\int \omega(t) d|\mu|$ is finite. We are mainly interested in the case that $L^{1}(\omega)$ is an algebra under the usual convolution product $f * g(x)=\int_{\mathbf{R}^{+}} f(x-t) g(t) d t$. The modern study of these algebras began with the fundamental paper of Graham Allan [A], which circulated as a preprint for several years before it was published.

By now much is known about such algebras. Many of the earlier results can be found in [D, section 4.7]. At first the results were proven for continuous weights, but this seems to be too strong a restriction since many important weights, like the norms of strongly continuous groups of linear operators, need not be continuous. We want to look at the

2010 Mathematics Subject Classification: Primary 43A20; Secondary 43A10.

Key words and phrases: convolution algebra, weight, equivalent weights.

The paper is in final form and no version of it will be published elsewhere. 
following question: For which weights $\omega$ are the results proved for special weights still valid? Essentially, we need to show that the same tools, such as weak* topologies, approximate identities, multiplier algebras, etc., available for the unweighted case of $L^{1}\left(\mathbf{R}^{+}\right)$are available for more general $L^{1}(\omega)$. We will largely survey results beginning with those in [Gr1], but we will give some new proofs and will also discuss some new results.

We will concentrate on three general questions. In Section 2 we will describe precisely for which weights $\omega$ we have that $L^{1}(\omega)$ is an algebra, and therefore a Banach algebra under an equivalent norm. In section 3 we will describe a class of weights, which we will call algebra weights, that have the same tools available isometrically as does $L^{1}\left(\mathbf{R}^{+}\right)$. In section 4 we will see that whenever $L^{1}(\omega)$ is an algebra there is an algebra weight $\omega^{\prime}$ for which $L^{1}(\omega)=L^{1}\left(\omega^{\prime}\right)$ with an equivalent norm and that, with careful definitions, we can adapt the same tools, but not necessarily isometrically, from $L^{1}(\omega)$ to $L^{1}\left(\omega^{\prime}\right)$. We will also often need to prove analogous results for translation invariance that we do for algebra structure and to study the relation between the algebra structure and translation invariance.

2. $L^{1}(\omega)$ as an algebra. In this section we describe exactly which weights $\omega$ make $L^{1}(\omega)$ an algebra. We also discuss the analogous simpler problem for the right translation operators $S_{a} f(x)=f(x-a)=\delta_{a} * f(x)$, where $\delta_{a}$ is the point mass at $a$. Since $L^{1}(\omega)$ is continuously embedded in $L_{l o c}^{1}\left(\mathbf{R}^{+}\right)$, the Fréchet algebra of locally integrable functions on $\mathbf{R}^{+}$, it follows that linear and bilinear operators continuous on $L_{l o c}^{1}\left(\mathbf{R}^{+}\right)$, such as convolution and translations, are continuous when defined on any $L^{1}(\omega)$. In particular, whenever $L^{1}(\omega)$ is an algebra, then convolution is a bounded bilinear operator so that $L^{1}(\omega)$ is a Banach algebra under an equivalent norm. The results in this section are adapted from [Gr1, section 2], so we will refer to this paper for the details of some proofs.

A standard argument shows that $L^{1}(\omega)$ is an algebra if there is a positive constant $K$ for which $\omega(x+y) \leq K \omega(x) \omega(y)$ for almost every $(x, y)$ in the first quadrant. Moreover, $\|f * g\| \leq K\|f\|\|g\|$ for the same constant $K$. Our main result is that the converse is also true. The following definitions provide the key techniques of the proof.

Definition 2.1. The number $a \geq 0$ is a right Lebesgue point of the locally integrable function $f$ if

$$
\lim _{h \rightarrow 0^{+}} \frac{1}{h} \int_{a}^{a+h}|f(x)-f(a)| d x=0 .
$$

It follows from the Lebesgue differentiation theorem that almost every number is a right Lebesgue point. It is also easy to see that if $a$ is a right Lebesgue point of $f$, then $(1 / h) \int_{0}^{h} f(a+x) d x$ has limit $f(a)$ as $h \rightarrow 0^{+}$.

Definition 2.2. The function $e_{h}$, for $h>0$, is defined to be $1 / h$ times the characteristic function of the interval $[0, h)$.

TheOrem 2.3 ([Gr1, Lem. (2.4), p. 534]). Suppose that $\omega$ is a weight and that $K$ is a positive number. Then the following are equivalent:

(a) $\|f * g\|_{\omega} \leq K\|f\|_{\omega}\|g\|_{\omega}$ for all $f$ and $g$ in $L^{1}(\omega)$.

(b) $\omega(x+y) \leq K \omega(x) \omega(y)$ for almost every $(x, y)$.

(c) $\omega(x+y) \leq K \omega(x) \omega(y)$ whenever $x, y$, and $x+y$ are all right Lebesgue points of $\omega$. 
The crux of the proof is to show that (a) implies (c). One considers the functions $f_{h}=\delta_{a} * e_{h}$ and $g_{h}=\delta_{b} * e_{h}$, where $a, b$, and $a+b$ are all right Lebesgue points of $\omega$. It follows from the comments after definition 2.1 that $\left\|f_{h}\right\| \rightarrow \omega(a)$ as $h \rightarrow 0^{+}$, and similarly for $g_{h}$. With more work one can also show that $\left\|f_{h} * g_{h}\right\|$ goes to $\omega(a+b)$. This shows that (a) implies (c).

As we pointed out above, if $L^{1}(\omega)$ is an algebra then there is a constant $K>0$ for which $\|f * g\|_{\omega} \leq K\|f\|_{\omega}\|g\|_{\omega}$ for all $f$ and $g$ in $L^{1}(\omega)$. In other words, $L^{1}(K \omega)$ is a Banach algebra with the same elements as $L^{1}(\omega)$. Hence we have the following corollary of Theorem 2.3.

COROLlary 2.4. For any weight $\omega$, the following are equivalent.

(a) $L^{1}(\omega)$ is an algebra.

(b) $L^{1}(\omega)$ is a Banach algebra under an equivalent norm.

(c) There is a $K \geq 0$ for which $\omega(x+y) \leq K \omega(x) \omega(y)$ for almost every $(x, y)$.

The analogue of Corollary 2.4 for the right translation operator $S_{a} f(x)=\delta_{a} * f(x)$ is much more elementary.

THEOREM 2.5. The Banach space $L^{1}(\omega)$ is invariant under right translation by a if and only if $\omega(x+a) / \omega(x)$ is essentially bounded. Moreover when these conditions hold the right translation operator is a bounded linear operator with norm

$$
\left\|S_{a}\right\|=\operatorname{ess} \cdot \sup \omega(x+a) / \omega(x) .
$$

Any almost everywhere inequality between locally integrable functions is easily seen to hold wherever all the functions have right Lebesgue points. Thus we obtain from the above theorem that:

$$
\omega(x+a) \leq\left\|S_{a}\right\| \omega(x),
$$

whenever both $x$ and $x+a$ are right Lebesgue points of $\omega$ [Gr1, Lemma (2.8) (D), p. 537]. Proof of Theorem 2.5. For each $f$ in $L^{1}(\omega)$, that is for each $f(x) \omega(x)$ in $L^{1}\left(\mathbf{R}^{+}\right)$, we have that the quantity

$$
\begin{aligned}
\left\|S_{a} f\right\|_{\omega} & =\int_{a}^{\infty}\left|S_{a} f(x)\right| \omega(x) d x=\int_{0}^{\infty}|f(x)| \omega(x+a) d x \\
& =\int_{0}^{\infty}|f(x) \omega(x)|(\omega(x+a) / \omega(x)) d x
\end{aligned}
$$

is finite for all such $f$ if and only if $\omega(x+a) / \omega(x)$ is essentially bounded. Moreover the sup of the integrals over all $f \omega$ of norm 1 in $L^{1}\left(\mathbf{R}^{+}\right)$is the essential supremum, that is the $L^{\infty}$ norm, of $\omega(x+a) / \omega(x)$. This completes the proof of the theorem.

We want to extend important known results from $L^{1}\left(\mathbf{R}^{+}\right)$to more general $L^{1}(\omega)$. The following definition gives us a useful tool for this purpose.

Definition 2.6. We let $L_{0}$ be the vector space of (equivalence classes of) integrable functions with bounded support on $\mathbf{R}^{+}$. We let $L_{0}(\omega)$ be this space with the norm inherited from $L^{1}(\omega)$.

The space $L_{0}$ is a dense subspace of each $L^{1}(\omega)$, but the norms on different $L_{0}(\omega)$ need not be equivalent. On the other hand, since we require both $\omega$ and $1 / \omega$ to be bounded on 
finite intervals, any sequence in $L^{1}(\omega)$ whose elements all have support in a fixed bounded interval will converge in $L^{1}(\omega)$ if and only if the sequence converges in $L^{1}\left(\mathbf{R}^{+}\right)$. We will need the following well-known elementary result on extending pointwise convergence of operators from a dense subspace of a Banach space to the whole space.

THEOREM 2.7. Suppose that $V$ is a dense subspace of the Banach space $X$ and that $\left\{S_{n}\right\}$ is a norm bounded sequence of linear operators from $X$ to the Banach space $Y$.

(a) If $T$ is a bounded linear operator from $X$ to $Y$ and $S_{n} x$ converges to Tx for all $x$ in $V$, then $S_{n} x$ converges to $T x$ for all $x$ in $X$.

(b) If $S_{n} x$ converges for all $x$ in $V$, then it converges for all $x$ in $X$ and the limit is a bounded linear operator.

We now apply $L_{0}$ and Theorem 2.7 to right translations on $L^{1}(\omega)$.

THEOREM 2.8. If $L^{1}(\omega)$ is invariant under right translations, then the right-translation semigroup is strongly continuous on $L^{1}(\omega)$ on $(0, \infty)$. It is strongly continuous at 0 if and only if $\left\|S_{t}\right\|$ is bounded for $t$ near 0 .

Proof. Suppose that the sequence $\left\{a_{n}\right\}$ in $\mathbf{R}^{+}$has limit $a$. Fix a function $f$ in $L_{0}$. Since the right-translation semigroup is strongly continuous on $L^{1}\left(\mathbf{R}^{+}\right)$, the sequence $S_{a_{n}} f$ converges to $S_{a} f$ in the usual $L^{1}$ norm. Since $f$ has bounded support, there is a fixed interval $[0, b]$ which contains the support of all the $S_{a_{n}} f$. Hence $S_{a_{n}} f$ converges to $S_{a} f$ in the norm of $L^{1}(\omega)$.

We just need to check that the sequence $\left\{S_{a_{n}}\right\}$ is bounded in norm. If we let $W(t)=$ $\left\|S_{t}\right\|$, then the weight is submultiplicative. Hence $W(t)$ is locally bounded on $(0, \infty)$ (see [D, Lemma 4.7.4, p. 521]) and is locally bounded on all of $\mathbf{R}^{+}$if it is bounded near 0 . The theorem now follows from Theorem 2.7(a) above.

For other proofs of the above theorem, see the references in the proof of [Gr1, Lemma $(2.8)(\mathrm{C})$, p. 537]. We close this section by relating translation invariance to $L^{1}(\omega)$ being an algebra.

THEOREM 2.9. If $L^{1}(\omega)$ is an algebra, then it is invariant under right translations. Moreover the right-translation semigroup is strongly continuous on $L^{1}(\omega)$.

Proof. We start by choosing $K$ as in Theorem 2.3, so that $\omega(x+y) \leq K \omega(x) \omega(y)$ for almost every $(x, y)$. We now first show that $L^{1}(\omega)$ is invariant under translation by $a$ when $a$ is a fixed right Lebesgue point of $\omega(x)$. For this fixed $a$, we have for almost every $x$ that both $x$ and $a+x$ are right Lebesgue points. Since $a$ is also a right Lebesgue point, it follows from Theorems $2.3(\mathrm{c})$ and 2.5 that $S_{a}$ is bounded with $\left\|S_{a}\right\| \leq K \omega(a)$. Since $\omega(x)$ is bounded on all intervals $[0, b]$, there is an $M$, which depends on $b$, for which $\left\|S_{x}\right\| \leq M$ for all right Lebesgue points $x$ in $[0, b]$.

Now suppose that $a$ is an arbitrary nonnegative number. Since almost every number is a right Lebesgue point of $\omega$, the right Lebesgue points form a dense subset of $\mathbf{R}^{+}$. Hence we can find a sequence $\left\{a_{n}\right\}$ of right Lebesgue points with limit $a$. Let $f$ be a function in $L_{0}$. Since the right-translation semigroup is strongly continuous on $L^{1}\left(\mathbf{R}^{+}\right)$, $S_{a_{n}} f$ converges to $S_{a} f$ in the norm of $L^{1}(\mathbf{R})$. Since $f$ has bounded support, it follows, just as in the previous proof, that the sequence $\left\{S_{a_{n}} f\right\}$ converges in the norm of $L^{1}(\omega)$ for 
all $f$ in $L_{0}$. It now follows from Theorem 2.7(b) that there is a bounded linear operator $T$ on $L^{1}(\omega)$ for which the sequence $\left\{S_{a_{n}} f\right\}$ converges strongly to $T f$ in $L^{1}(\omega)$. Since $L^{1}(\omega)$ is continuously imbedded in the algebra of locally integrable functions on $\mathbf{R}^{+}$, the sequence $\left\{S_{a_{n}} f\right\}$ converges to $T f$ in $L_{l o c}^{1}\left(\mathbf{R}^{+}\right)$for all $f$ in $L^{1}(\omega)$. But $S_{a_{n}} f$ converges to $S_{a} f$ in $L_{l o c}^{1}\left(\mathbf{R}^{+}\right)$. Hence $S_{a}=T$, which is a bounded operator on $L^{1}(\omega)$. This proves right-translation invariance.

We showed above that on any interval $[0, b]$, the norms of all right translations with respect to a right Lebesgue point are bounded by some constant $M$. Since every right translation $S_{a}$ is a strong limit of right translations by right Lebesgue points, it follows that these too are bounded by $M$. So $\left\|S_{a}\right\| \leq M$ for all $a$ in $[0, b]$. It now follows from Theorem 2.8 that the right-translation semigroup is strongly continuous on $L^{1}(\omega)$.

For a different proof of right-translation invariance, see the paragraph after the proof of [Gr1, Lemma (3.3), p. 539].

3. Isometrically good weights. In this section, we will describe a class of weights for which $L^{1}(\omega)$ is an algebra for which we have isometrically the same basic harmonic analysis tools that we have for $L^{1}\left(\mathbf{R}^{+}\right)$. This was done for continuous weights by Ghahramani [Gh, section 1] (see also [D, section 4.7]). We will describe results from [Gr2] and $[\mathrm{W}]$ that replace continuity by right continuity. In the next section we will show how to extend these results, but not isometrically, to the case that $L^{1}(\omega)$ is an algebra. This will be done by first showing that there is a good weight $\omega^{\prime}$, of the type we consider in this section, for which $L^{1}\left(\omega^{\prime}\right)=L^{1}(\omega)$. Continuity is too restrictive precisely because there need not be a continuous weight $\omega^{\prime}$ with $L^{1}\left(\omega^{\prime}\right)=L^{1}(\omega)$.

One of the main tools in harmonic analysis is the measure algebra and its relation to the $L^{1}$ algebra. So we will need to know that $M(\omega)$ is an algebra. Since $M(\omega)$ is continuously imbedded in the convolution algebra of locally finite measures on $\mathbf{R}^{+}$, convolution multiplication on $M(\omega)$ will be a bounded bilinear operator whenever $M(\omega)$ is an algebra. For $M(\omega)$, unlike $L^{1}(\omega)$, we will need our inequalities to hold everywhere instead of just almost everywhere, because the formula $\left\|\delta_{a+b}\right\| \leq K\left\|\delta_{a}\right\|\left\|\delta_{b}\right\|$ translates to the inequality $\omega(a+b) \leq K \omega(a) \omega(b)$. We then obtain the following analogue of Theorem 2.3 and Corollary 2.4. The result is essentially [Gr4, Theorem 2.5 (i)(ii), p. 412].

THEOREM 3.1. For any weight $\omega$ and any positive number $K$, the following conditions are equivalent. Moreover there is a positive $K$ for which the conditions hold, if and only if $M(\omega)$ is an algebra.

(a) $\omega(x+y) \leq K \omega(x) \omega(y)$ for all nonnegative $x$ and $y$.

(b) $\|\mu * \nu\|_{\omega} \leq K\|\mu\|_{\omega}\|\nu\|_{\omega}$ for all $\mu$ and $\nu$ in $M(\omega)$.

(c) $M(K \omega)$ is a Banach algebra.

Of course $M(K \omega)$ is the same space as $M(\omega)$ but with the equivalent norm $\|\mu\|_{K_{\omega}}=$ $K\|\mu\|_{\omega}$. Any inequality for functions that holds almost everywhere will hold in particular at all points of right continuity. Thus when the weight $\omega$ is right continuous, condition (a) in the above theorem is equivalent to condition (b) in Theorem 2.3 above. Thus for right continuous weights, $L^{1}(\omega)$ is an algebra if and only if $M(\omega)$ is an algebra. Moreover 
they are Banach algebras if and only if the constant $K=1$, that is if and only if the weight $\omega$ is submultiplicative. If we want isometric results, we will need that the identity $\delta_{0}$ has norm 1 , that is we must have $\omega(0)=1$. These conditions are enough to give us the class of weights we want [Gr2, Def. 1.1, p.590].

Definition 3.2. The weight $\omega(x)$ on $\mathbf{R}^{+}$is an algebra weight if $\omega$ is submultiplicative, right continuous, and has $\omega(0)=1$.

In order to be able to give $M(\omega)$ a weak* topology, we need a predual. When $\omega$ is continuous this is easily done [Gh] using the Riesz representation theorem. For right continuous weights Willis [W, p. 303] uses the following space.

Definition 3.3. When $\omega(x)$ is right continuous, we let $C_{0}(1 / \omega)$ be the space of continuous functions $f(x)$ on $\mathbf{R}^{+}$for which $f(x) / \omega(x)$ is bounded and has limit 0 as $x$ goes to $\infty$. We give $C_{0}(1 / \omega)$ the weighted sup norm $\|f\|=\sup |f(x) / \omega(x)|$.

We are now ready to state our major result about algebra norms. The theorem below is essentially [Gr2, Th. 2.2, p. 592]. Much of the result is adapted from [W], particularly by adding isometric results. The functions $e_{h}$ in the theorem are defined in definition 2.2 above.

THEOREM 3.4. Suppose that $\omega$ is an algebra weight. Then both $L^{1}(\omega)$ and $M(\omega)$ are Banach algebras for which the following properties hold.

(a) The functions $\left\{e_{h}\right\}$ are a bounded approximate identity as $h$ goes to 0 , with $\lim _{h \rightarrow 0^{+}}\left\|e_{h}\right\|=1$.

(b) The right translation operator $S_{a} f(x)=\delta_{a} * f(x)$ has operator norm $\omega(a)$.

(c) The Banach space $M(\omega)$ is isometrically isomorphic to the dual space of $C_{0}(1 / \omega)$ when we identify the measure $\mu$ with the linear functional $\langle\mu, f\rangle=\int_{\mathbf{R}^{+}} f(x) d \mu$.

(d) The algebra $M(\omega)$ is isometrically isomorphic to the multiplier algebra of $L^{1}(\omega)$ when we identify the measure $\mu$ with the operation of convolution by $\mu$ on $L^{1}(\omega)$.

We only give proofs of those parts that are different from the proofs, or omitted from the proofs, in [Gr2, Th. 2.2, p. 592] and the references there. In particular we omit the proof of (c) which adds the isometric result to Willis's proof of [W, Lem. 1.2, p. 303].

Proof. Since $\omega$ is right continuous it follows from the elementary fundamental theorem of calculus that $\left\|e_{h}\right\|_{\omega}$ goes to $\omega(0)=1$ as $h$ goes to $0^{+}$. In particular $\left\|e_{h}\right\|_{\omega}$ is bounded for $h$ near 0 . Since $\left\{e_{h}\right\}$ is a bounded approximate identity in $L^{1}\left(\mathbf{R}^{+}\right)$we have, for all $f$ in $L_{0}$, that $e_{h} * f$ goes to $f$ in the $L^{1}$ norm. But the supports of the $e_{h} * f$ for small $h$ all lie in a single bounded interval. Hence $e_{h} * f$ goes to $f$ as $h$ goes to 0 in the norm of $L^{1}(\omega)$ as well. Since the norms of the $e_{h}$ are bounded and $L_{0}$ is a dense subspace of $L^{1}(\omega)$, this tells us that $\left\{e_{h}\right\}$ is a bounded approximate identity for $L^{1}(\omega)$. This proves (a).

Since $\omega$ is submultiplicative, it follows from Theorem 2.5 that $\left\|S_{a}\right\| \leq \omega(a)$. On the other hand it follows from the fundamental theorem of calculus that $\left\|S_{a}\left(e_{h}\right)\right\|_{\omega}$ goes to $\omega(a)$ as $h$ goes to 0 , and we have already observed that $\left\|e_{h}\right\|_{\omega}$ goes to 1 . Hence we also have $\left\|S_{a}\right\| \geq \omega(a)$. This completes the proof of $(\mathrm{b})$. 
We will not repeat either the proof of (c) or the calculation of the norm of convolution with $\mu$ that is given in [Gr2, Th. 2.2, p. 592]. Every proof we have seen in print, even for the case of continuous weights, of the fact that all multipliers of $L^{1}(\omega)$ are given by convolution with a measure in $M(\omega)$, simply points out that the analogous proof for locally compact groups or for some other analogue of $M(\omega)$ carries through without change. We will therefore provide the details here.

Suppose that $T$ is a multiplier of $L^{1}(\omega)$. Let $\left\{h_{n}\right\}$ be a bounded approximate identity of $L^{1}(\omega)$. Then $\left\{T h_{n}\right\}$ is a bounded sequence in $L^{1}(\omega) \subseteq M(\omega)$. Since $M(\omega)$ is the dual space of a separable Banach space, bounded subsets are precompact and metrizable in the weak* topology and therefore the sequence $\left\{h_{n}\right\}$ has a subsequence $\left\{h_{n}^{\prime}\right\}$ for which the sequence $\left\{T h_{n}^{\prime}\right\}$ has a weak* limit $\mu$ in $M(\omega)$. We will show that $T f=\mu * f$ for all $f$ in $L^{1}(\omega)$. Since $T$ is a multiplier, all $\left(T h_{n}^{\prime}\right) * f=T\left(h_{n}^{\prime} * f\right)$. Convolution with a fixed element $f$ in $M(\omega)$ is weak* continuous [Gr2, p. 595] so $T h_{n}^{\prime} * f$ has weak ${ }^{*}$ limit $\mu * f$. On the other hand, $\left\{h_{n}^{\prime}\right\}$ is a bounded approximate identity, so $T\left(h_{n}^{\prime} * f\right)$ has norm limit $T(f)$ and therefore also has weak* limit $T(f)$. Thus we have that the multiplier $T$ of $L^{1}(\omega)$ is convolution with the measure $\mu$ in $M(\omega)$.

4. Equivalent weights. In this section we show how to extend the good properties we described in the previous section for $L^{1}(\omega)$ when $\omega$ is an algebra weight to the more general case, described in Theorem 2.3 above, where we just know that $L^{1}(\omega)$ is an algebra. The fundamental result, Theorem 4.2 below, says that if $L^{1}(\omega)$ is an algebra then we can always find an algebra weight $\omega^{\prime}$ with $L^{1}\left(\omega^{\prime}\right)=L^{1}(\omega)$. Recall that whenever $L^{1}\left(\omega^{\prime}\right)=L^{1}(\omega)$, these two Banach spaces have equivalent norms. This tells us that any result proved in the case of algebra weights remains true whenever $L^{1}(\omega)$ is an algebra, provided that the result just involves the norm topology or is just algebraic. We can lose isometric results, and we can have $M\left(\omega^{\prime}\right) \neq M(\omega)$ when $L^{1}\left(\omega^{\prime}\right)=L^{1}(\omega)$. These results are adapted from [Gr1] and [Gr2]. Later in this section we will describe newer results that let us adapt weak* results from the case of algebra weights to the general case where $L^{1}(\omega)$ is an algebra. We first need to describe the equality of two weighted spaces in terms of the weights involved.

Definition 4.1. Two weights $\omega_{1}$ and $\omega_{2}$ are said to be essentially equivalent if both $\omega_{1} / \omega_{2}$ and $\omega_{2} / \omega_{1}$ are essentially bounded. The weights are equivalent if these two ratios are bounded, not just essentially bounded.

Thus $\omega_{1}$ and $\omega_{2}$ are essentially equivalent if and only if $L^{1}\left(\omega_{1}\right)=L^{1}\left(\omega_{2}\right)$ and are equivalent if and only if $M\left(\omega_{1}\right)=M\left(\omega_{2}\right)$. If $\omega_{1}$ and $\omega_{2}$ are both right continuous, they are equivalent if and only if they are essentially equivalent. We are now ready for our major result on equivalence of weights.

THEOREM 4.2 ([Gr2, Theorem 2.1, p. 591]). If $L^{1}(\omega)$ is an algebra, then there is an algebra weight $\omega^{\prime}$ which is essentially equivalent to $\omega$. That is, $L^{1}\left(\omega^{\prime}\right)=L^{1}(\omega)$ with equivalent norms.

We know from Theorem 2.9 that when $L^{1}(\omega)$ is an algebra, then it is right-translation invariant and the right-translation semigroup is strongly continuous. The most substantial 
part of the proof of Theorem 4.2 is the case where we just assume $L^{1}(\omega)$ has a strongly continuous right-translation semigroup.

Theorem 4.3 ([Gr1, Th. (3.1) (A)(B), pp. 537-538]). Suppose that $L^{1}(\omega)$ is invariant under right translations. If the right-translation semigroup is strongly continuous, then there is a right continuous weight $\omega_{1}$ which is essentially equivalent to $\omega$ (so $L^{1}\left(\omega_{1}\right)=$ $L^{1}(\omega)$ with equivalent norms). Moreover we can choose $\omega_{1}$ so that there is a real number $r$ for which $\omega_{1}(x) / e^{r x}$ is weakly decreasing.

We start with the proof of Theorem 4.2, adapted from [Gr4, Lem. 2.7, p. 413], assuming Theorem 4.3 .

Proof of Theorem 4.2. Since nothing essential is changed by multiplying or dividing by $e^{r x}$ we can assume that $\omega_{1}$ is weakly decreasing. Since $L^{1}\left(\omega_{1}\right)$ is an algebra and $\omega_{1}$ is right continuous the condition $\omega_{1}(x+y) \leq K \omega_{1}(x) \omega_{1}(y)$ of Corollary 2.4(c) holds for all $x$ and $y$. Then the weight $K \omega_{1}(x)$ is a weakly decreasing right continuous submultiplicative weight equivalent to $\omega_{1}$ and therefore essentially equivalent to $\omega$. Since $K \omega_{1}(x)$ is submultiplicative, it is easy to see that $K \omega_{1}(0) \geq 1$. Finally, we let $\omega^{\prime}(x)=\min \left(1, K \omega_{1}(x)\right)$. It is now easy to check that $\omega^{\prime}(x)$ is an algebra weight equivalent to $K \omega_{1}(x)$ and therefore essentially equivalent to $\omega(x)$.

Proof of Theorem 4.3. Let $W(x)=\left\|S_{x}\right\|$. Since $S_{x}$ is a semigroup, it follows that $W(x)$ is submultiplicative. Hence $W(x)^{1 / x}$ has a finite limit as $x$ goes to infinity. Hence there is an $r$ for which $W(x) / e^{r x}$ is bounded. Since $\omega_{1}(x) / e^{r x}$ is essentially equivalent to $\omega(x) / e^{r x}$ if and only if $\omega_{1}(x)$ is essentially equivalent to $\omega(x)$, we can assume without loss of generality that $W(x)$ is bounded, say $W(x) \leq M$ for all $x$. It then follows from formula (2.1) that if $b$ is a right Lebesgue point of $\omega$, then $\omega(x) \leq M$. Therefore $\omega(x) \leq M$ for almost every $x \geq b$. Hence the weight $\omega(x)$ will be essentially bounded under our assumption that $W(x)$ is bounded.

We now define $\omega_{1}(x)=$ ess.sup. $\{\omega(t): t \geq x\}$. It is clear that $\omega_{1}(x)$ is weakly decreasing and right continuous and that $\omega(x) \leq \omega_{1}(x)$ at every right Lebesgue point of $\omega$, and therefore almost everywhere. Conversely, if $x$ is a right Lebesgue point of $\omega$ then it follows from formula (2.1) again that $\omega(t) \leq M \omega(x)$ for almost every $t$ greater than $x$. Thus $\omega_{1}(x) \leq M \omega(x)$ for almost every $x$. Hence $\omega_{1}$ is essentially equivalent to $\omega$ and the proof is complete.

For many years, it appeared that Theorems 3.4 and 4.2 together (which are adapted from [Gr1] and [Gr2]) were adequate to prove results valid for the general case where $L^{1}(\omega)$ is just assumed to be an algebra. One could even use the weak ${ }^{*}$ topology and other tools for algebra weights from Theorem 3.4, as long as the final result involved only the norm topology. More recently, some of the most striking results involve the weak* topology directly (see for instance [Gr3] and [Gr5]). The problem is that we do not have $M\left(\omega^{\prime}\right)=M(\omega)$ unless $\omega$ and $\omega^{\prime}$ are equivalent and not just essentially equivalent. We will now describe results, adapted from [Gr6], which describe a measure algebra which is both a dual space containing $L^{1}(\omega)$ and the multiplier algebra of the algebra $L^{1}(\omega)$. This will require us to first adapt Definition 3.3 to weights that need not be right continuous. 
Definition 4.4. If $\omega(x)$ is a weight, we let $C_{0}(1 / \omega)$ be the space of continuous functions $f(x)$ on $\mathbf{R}^{+}$for which $f(x) / \omega(x)$ is essentially bounded and has essential limit 0 as $x$ goes to infinity. We give $C_{0}(1 / \omega)$ the norm it inherits as a subspace of $L^{\infty}(1 / \omega)$. That is, $\|f\|=\operatorname{ess} \cdot \sup |f(x) / \omega(x)|$.

When $\omega$ is right continuous, the above definition agrees with Definition 3.3. If $\omega^{\prime}$ is any weight essentially equivalent to $\omega$, then $C_{0}\left(1 / \omega^{\prime}\right)=C_{0}(1 / \omega)$ with the same norm.

TheOREM 4.5 ([Gr6, Th. 4.1]). Suppose that $L^{1}(\omega)$ is an algebra and let $\omega^{\prime}$ be an algebra weight essentially equivalent to $\omega$. Then we have the following:

(a) $M\left(\omega^{\prime}\right)$ is the dual space of $C_{0}(1 / \omega)$, with an equivalent norm, when we identify the measure $\mu$ in $M\left(\omega^{\prime}\right)$ with the linear functional $\langle\mu, f\rangle=\int_{\mathbf{R}^{+}} f(x) d \mu$ on $C_{0}(1 / \omega)$.

(b) $M\left(\omega^{\prime}\right)$ is the multiplier algebra of $L^{1}(\omega)$, with an equivalent norm, when we identify the measure $\mu$ with the operation of convolution by $\mu$ on $L^{1}(\omega)$.

Proof. Since $\omega^{\prime}$ is essentially equivalent to $\omega$, we have that $L^{1}\left(\omega^{\prime}\right)=L^{1}(\omega)$ with equivalent norms and $C_{0}\left(1 / \omega^{\prime}\right)=C_{0}(1 / \omega)$ with the same norm. But we know from Theorem 3.4 that $M\left(\omega^{\prime}\right)$ is the dual space of $C_{0}\left(1 / \omega^{\prime}\right)=C_{0}(1 / \omega)$ and the multiplier algebra of $L^{1}\left(\omega^{\prime}\right)=$ $L^{1}(\omega)$. This completes the proof.

Since right continuous weights that are essentially equivalent to each other are actually equivalent to each other, the algebra $M\left(\omega^{\prime}\right)$ is unchanged if $\omega^{\prime}$ is replaced by another right continuous weight essentially equivalent to $\omega$, and hence equivalent to $\omega^{\prime}$. Since $M\left(\omega^{\prime}\right)$ is the multiplier algebra of $L^{1}(\omega)$, it is clear that $L^{1}(\omega) \subseteq M\left(\omega^{\prime}\right)$, but not isometrically. When $M(\omega)$ is an algebra it contains $L^{1}(\omega)$ as a closed ideal, so we have $L^{1}(\omega) \subseteq M(\omega) \subseteq$ $M\left(\omega^{\prime}\right)$. If $M(\omega)$ is not an algebra, then $M(\omega)$ must be different from the algebra $M\left(\omega^{\prime}\right)$. I had originally thought [Gr4, Th. 2.5 (iii), p. 412] that whenever $M(\omega)$ was an algebra, then there was an algebra weight $\omega^{\prime}$ equivalent to $\omega$ so that $M\left(\omega^{\prime}\right)=M(\omega)$. But this result is false; there is an example in [Gr6] of a weight $\omega$ for which $M(\omega)$ is an algebra but $\omega$ is not equivalent to any right continuous weight. For this example $M\left(\omega^{\prime}\right) \neq M(\omega)$.

I do not know which submultiplicative weights are equivalent, and not just essentially equivalent, to what we have called algebra weights in Definition 3.2. Fortunately the most important algebra weights, those that come from semigroups, are equivalent to algebra weights. This was proved on an ad hoc basis, whenever it was needed, in several papers. We give the details below.

We say that $\omega(t)$ is a semigroup weight if there is a strongly continuous semigroup $T_{t}$ on some Banach space $E$ with $\omega(t)=\left\|T_{t}\right\|$.

THEOREM 4.6. If $\omega(t)$ is a semigroup weight, then there is an algebra weight $\omega^{\prime}$ with $M\left(\omega^{\prime}\right)=M(\omega)$.

Proof. Suppose that $\omega(t)$ is the semigroup weight given by the semigroup $T_{t}$ on the Banach space $E$. By replacing $T_{t}$ with an appropriate $T_{t} / e^{r t}$ we can assume without loss of generality that $T_{t}$ is a bounded semigroup. When $T_{t}$ is a bounded semigroup on a Banach space $E$, it is a standard result that one can find an equivalent norm on $E$ under which $T_{t}$ becomes a contraction semigroup (See for instance [BD, Lem. 3, pp. 90-91]). When the norm on $E$ is replaced by an equivalent norm, the new operator norm on $B(E)$ 
is also equivalent to the old operator norm. So if we let $\omega^{\prime}(t)=\left\|T_{t}\right\|^{\prime}$, where the prime designates the new equivalent operator norm, we have that $\omega^{\prime}$ is equivalent to $\omega$. Since the semigroup $T_{t}$ is strongly continuous, the semigroup norm $\omega^{\prime}(t)$ is a supremum of continuous functions and is therefore lower semicontinuous. Since the semigroup $T_{t}$ is a contraction semigroup under the new norm, we also have that $\omega^{\prime}(t)$ is weakly decreasing. Since $\omega^{\prime}(t)$ is both lower semicontinuous and weakly decreasing, it is also right continuous. Hence $\omega^{\prime}(t)$ is an algebra norm equivalent to our original semigroup norm $\omega(t)$. This completes the proof.

Now we know that if $\omega$ is a semigroup weight, then there is an algebra weight $\omega^{\prime}$ for which we not only have $L^{1}\left(\omega^{\prime}\right)=L^{1}(\omega)$ and $C_{0}\left(1 / \omega^{\prime}\right)=C_{0}(1 / \omega)$ but also $M\left(\omega^{\prime}\right)=M(\omega)$. Thus we have the following useful result.

COROLlary 4.7. If $\omega(t)$ is a semigroup weight, then all the results given for algebra weights in Theorem 3.4 remain true, but not necessarily isometrically, for $\omega(t)$.

Actually, the condition in Theorem 4.6 characterizes weights equivalent to algebra weights.

COROLlarY 4.8. The weight $\omega(t)$ is equivalent to an algebra weight $\omega^{\prime}(t)$ if and only if $\omega(t)$ is a semigroup weight.

Proof. We still need to show that if a weight is equivalent to an algebra weight, then the original weight is a semigroup weight. It will be enough to show that every algebra weight is a semigroup weight. But Theorem 3.4(b) shows that an algebra weight is a semigroup norm for the semigroup of right translations. This completes the proof.

In a technical sense, Corollary 4.8 answers the question of which weights are equivalent, and not just essentially equivalent, to an algebra weight. This answer really is not adequate, because I know of no direct way to determine if a given weight $\omega(t)$ is a semigroup weight.

Acknowledgements. This paper is based on a lecture delivered at the $19^{\text {th }}$ International Conference on Banach Algebras held at Będlewo, July 14-24, 2009. The support for the meeting by the Polish Academy of Sciences, the European Science Foundation under the ESF-EMS-ERCOM partnership, and the Faculty of Mathematics and Computer Science of the Adam Mickiewicz University at Poznan is gratefully acknowledged.

\section{References}

[A] G. R. Allan, Ideals of rapidly growing functions, in: Proc. Int. Symp. on Functional Analysis (Forum for Functional Analysis and Its Applications), Ibadan, 1977.

[BD] F. F. Bonsall and J. Duncan, Numerical Ranges of Operators on Normed Spaces and of Elements of Normed Algebras, London Math. Soc. Lecture Note Ser. 2, Cambridge Univ. Press, Cambridge, 1971.

[D] H. G. Dales, Banach Algebras and Automatic Continuity, London Math. Soc. Monographs (N.S.) 24, Oxford Univ. Press, Oxford, 2000.

[Gh] F. Ghahramani, Homomorphisms and derivations on weighted convolution algebras, J. London Math. Soc. (2) 21 (1980), 149-161. 
[Gr1] S. Grabiner, Weighted convolution algebras on the half line, J. Math. Anal. Appl. 83 (1981), 531-553.

[Gr2] S. Grabiner, Homomorphisms and semigroups in weighted convolution algebras, Indiana Univ. Math. J. 37 (1988), 589-615.

[Gr3] S. Grabiner, Weak ${ }^{*}$ properties of weighted convolution algebras, Proc. Amer. Math. Soc. 132 (2004), 1675-1684.

[Gr4] S. Grabiner, Equivalent weights and standard homomorphisms for convolution algebras on $\mathbf{R}^{+}$, Proc. Edinburgh Math. Soc. 52 (2009), 409-418.

[Gr5] S. Grabiner, Weak* properties of weighted convolution algebras II, Studia Math. 198 (2010), 53-67.

[Gr6] S. Grabiner, Corrigendum to "Equivalent weights and standard homomorphisms for convolution algebras on $\mathbf{R}^{+}$", Proc. Edinburgh Math. Soc. 53 (2010), 415-417.

[W] G. A. Willis, The continuity of derivations and module homomorphisms, J. Austral. Math. Soc. Ser. A 40 (1986), 299-320. 
